\title{
Biomarkers in multiple sclerosis: Role of antibodies
}

\author{
Thomas Berger* and Markus Reindl \\ Clinical Department of Neurology, Innsbruck Medical University, Innsbruck, Austria
}

\begin{abstract}
The first international workshop on "Biomarkers in Multiple Sclerosis" was organized by B. Bielekova, R. Hohlfeld, R. Martin and U. Utz from April 14-16, 2004, in Washington, DC. The workshop intended to discuss the current status and potential applicability of biological markers for the understanding of the pathogenesis, diagnosis, and therapy of multiple sclerosis. The present review summarizes the presentation on the potential role of antibodies as biomarkers for diagnosis, disease activity, classification and prediction of clinical courses in multiple sclerosis.
\end{abstract}

\section{Introduction}

Multiple sclerosis (MS) is the most common neurological disease in young adults with the risk of subsequent chronic functional impairment and disability after 10 to 15 years of disease duration [34]. MS is not a single inflammatory demyelinating central nervous system (CNS) disease, but a complex of disorders with heterogeneous clinical presentations, disease courses, neuro-pathological, immunological and neuro-radiological features.

MS is considered to be of autoimmune origin [20, 42] and neuro-pathologically characterized by variable extents of focal inflammation, demyelination, axonal damage, gliotic scarring and atrophy, but also by remyelination and regeneration in the CNS. Immunopathogenetic and disease modifying therapeutic concepts focused mainly on inflammation in MS. Briefly, autoreactive T-cells cross the blood-brainbarrier to recognize liberated myelin and non-myelin components in the perivascular space. The initial, locally restricted CNS inflammatory process is subsequently amplified by a huge network of adhesion molecules, proinflammatory cytokines, chemokines

*Corresponding author: Prof. Thomas Berger, MD, MSc, Clinical Department of Neurology, Innsbruck Medical University, Anichstrasse 35, A-6020 Innsbruck, Austria. Tel.: +43 512504 23860; Fax: +43 512504 24260; E-mail: thomas.berger@uibk.ac.at. and other cellular effector molecules. Antigen and epitope spreading contributes importantly to the propagation of inflammation [27]. However, in general, T-cells alone are not sufficient to produce the typical demyelinating plaques in the CNS white matter. Additional demyelinating amplification factors are required and recent work has suggested that these factors may differ between patients [30]. This leads to the concept of MS as a heterogeneous disease with respect to four pathogenetic mechanisms of demyelination [23,25,30, $31]$. One of these subtypes is characterized by features of antibody-mediated demyelination [44], another subtype by oligodendrocyte pathology resembling what is typically seen in acute stroke [26].

\section{The search for biological markers in multiple sclerosis}

The search for biological markers in accessible body fluids (cerebrospinal fluid [CSF], blood, urine) of MS patients has been a scientific focus over the past decades. Identification of such markers would be crucial to further understanding of the etio-pathogenesis of MS, as well as for diagnosis, rational design of treatment regimens and monitoring treatment effects.

With regard to different steps of the immunopathogenetic cascade [20,42] or different clinical disease stages (clinically isolated syndromes, relapsingremitting and chronic progressive MS) numerous bi- 


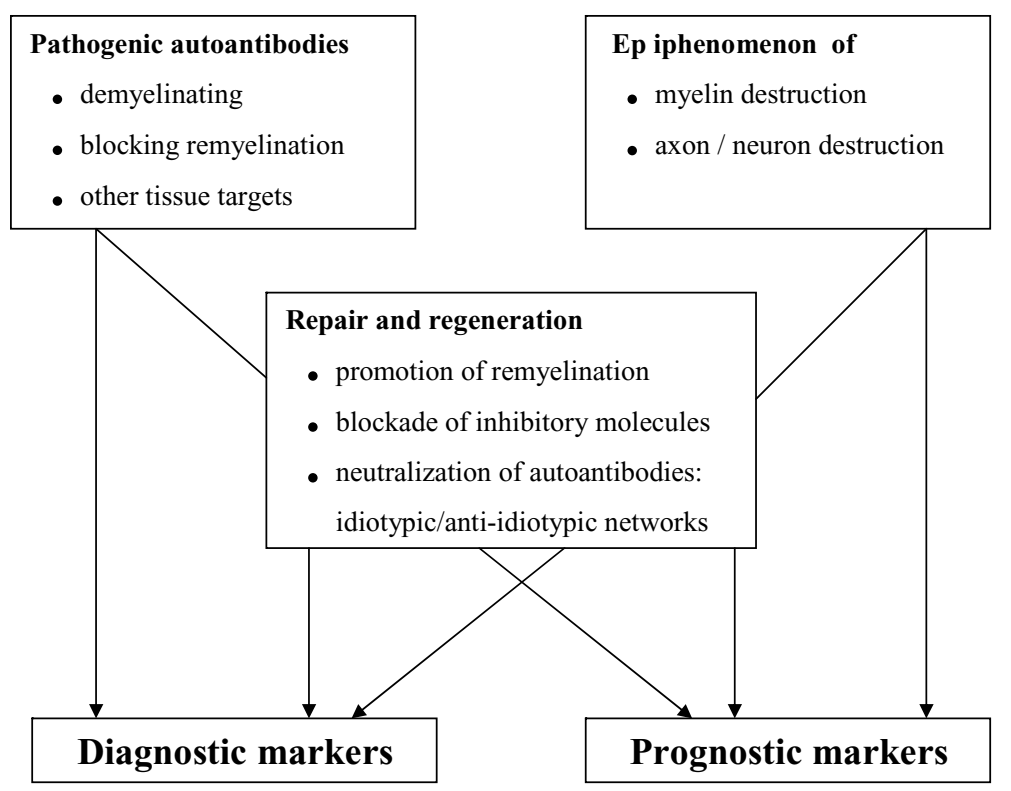

Fig. 1. Potential role of antibodies in multiple sclerosis.

ological marker candidates have been investigated in MS: inflammation (cytokines, chemokines, effector cell products), blood-brain-barrier dysfunction (adhesion molecules, matrix metalloproteases), demyelination (cytokines, antibodies, complement components, macrophage products), axonal destruction (neuronal proteins, oxidative stress, excitatoric amino acids), gliosis (biochemical markers) and remyelination (myelin products, adhesion molecules, neurotrophic factors).

However, most of the obtained research results on body fluid markers in MS have been either disappointingly negative or remain discussed controversially or still await confirmation.

\section{Autoantibodies in multiple sclerosis - general thoughts}

Autoantibodies may reflect the presence, nature, and intensity of a certain immune response [19]. Antibodies are therefore potential markers for diagnosis, disease activity, classification and prediction of clinical courses [8] - and already used in daily clinical practice for many neurological and other immune-mediated diseases. It is thus tempting to speculate that according to the clinical request (e.g. diagnosis, classification, prediction, monitoring of disease and therapy) detection of antibodies may provide different information in MS, depending on e.g. their target tissue specificity, biologi- cal activity, role in different stages of the immunopathogenetic cascade and time of occurrence/disappearance. To complicate these hypotheses antibodies may have different biological functions: some antibodies may be primarily involved at different stages of the immunopathogenetic cascade (e.g. demyelination), some may facilitate repair mechanisms (e.g. remyelination) and some may be part of a balancing natural autoimmunity system (Fig. 1).

\section{Autoantibodies as diagnostic markers in multiple sclerosis}

Since the seminal finding of elevated immunoglobulin (Ig), which mainly involves the IgG1 and IgG3 isotypes, in the CSF of more than $90 \%$ of MS patients [21], detection of oligoclonal Ig is an important diagnostic marker in MS [4,12,34]. Typically, and in contrast to acute infectious CNS diseases, the oligoclonal Ig response persists over time in MS. This pattern and the little clonotypic variation of oligoclonal bands in the CSF over time may be due to the fact that the focus of the humoral response does not change during the course of the disease. However, the dynamics of oligoclonal bands in the CSF of MS patients treated by disease modifying drugs has not been systematically studied so far. More importantly, the antigen-specificities of these oligoclonal Ig bands still remain to be defined. CSF oligoclonal bands do not necessarily represent au- 
toantibodies against neuronal proteins, but may also be directed against infectious agents, a finding which is not specific for MS. Many studies have tried to identify autoantibodies directed against various myelin and non-myelin target antigens in the serum and CSF of MS patients (Table 1). However, none of these studies revealed convincingly an MS specific antibody response against CNS antigens. Most antibodies detected in MS are also found in other neurological and systemic conditions as well as to a lower extent in healthy controls. There are several explanations for these findings [8]: (1) the causing auto-antigen(s) in MS is/are not identified so far, possibly due to the complex heterogeneity of MS. (2) the knowledge about the biological role of antibodies in MS is still scant, probably due to the biological complexity of antibodies ranging from a mere bystander phenomenon to pathogenic, regulatory and even reparative properties. (3) technical issues concerning different detecting systems with different antigen preparations add to the controversy of previous findings on autoantibodies in MS.

It is therefore to conclude that specific autoantibodies as diagnostic markers in MS are not available yet.

Recent neuro-pathological investigations, which revealed four subtypes of demyelination in MS, were the first milestone to distinguish immunopathogenetic MS subtypes - irrespective of the clinical phenotype [23, $25,30,31]$. Now there are promising studies that support the use of antibodies in classifying MS patients by immunological (and neuro-radiological) means. Antibodies directed to myelin oligodendrocyte glycoprotein (MOG), a highly immunogenic CNS specific protein of the outer myelin sheath, have been repeatedly shown in CSF and sera in a subset of MS patients and patients with other inflammatory neurological conditions [39]. In MS, these anti-MOG antibodies are present already in early MS (e.g. first demyelinating event) and accumulate together with other anti-myelin antibodies (e.g. against myelin basic protein, MBP) over time during disease progression. Anti-MOG IgM antibodies are the prominent Ig isotype; within the IgG subclass IgG1 and IgG3 isotypes predominate [14,39].

In addition, some of the anti-MOG IgM antibodies are able to fix complement and to activate the lytic terminal complement complex (Berger et al, unpublished). Patients with high titers of anti-MOG IgM antibodies may comprise an antibody-mediated subtype of MS [11] and seem to response to antibody-focused treatments, such as plasma exchange or intravenous immunoglobulin [14].

Another neuro-pathological subtype in MS shows characteristics of a dying-back oligodendro-gliopathy, which is also a neuro-pathological hallmark of stroke. A recent study revealed that MS brain biopsy tissue showed co-localization of hypoxia inducible factor alpha-1 and of a monoclonal antibody termed d110 exclusively in this subtype [26]. Originally, d110 had been identified as recognizing a surface epitope of canine distemper virus nucleocapsid. However, neither this virus nor viral remnants could be detected in the CNS tissue of MS patients. So the target antigen of the $\mathrm{d} 110$ antibody response remains to be determined. Nevertheless, it is possible to detect d110 in the CSF of MS patients. Further investigations are necessary to see whether this marker is useful either to identify patients with this specific neuro-pathological subtype of demyelination or to distinguish these patients from other immunopathogenetic subtypes.

\section{Autoantibodies as predictive markers in multiple sclerosis}

Treatment, management and counseling of MS patients would be markedly improved by the identification of reliable clinical, genetic, immunological or neuro-radiological prognostic markers. Research in the past decades sought to find negative or positive predictors regarding conversion to clinically definite MS after a first demyelinating event, to disease type and disease activity in terms of occurrence of future relapses and disease progression, and especially the time point of conversion to secondary progressive MS. Unfortunately, most of the results to date merely demonstrate associations with certain disease issues, but failed to provide reliable biological markers of predictive value in individual patients.

In patients with a first demyelinating event, the conversion to clinically definite MS is unpredictable and long observation periods and repetitive MRI investigations are needed. Recently, anti-myelin antibodies were shown to be able to predict the risk of a first relapse in patients with a clinically isolated syndrome (CIS) suggestive of MS [9]. In this active CIS cohort (as demonstrated by positive MRI findings and oligoclonal bands in CSF) patients seropositive for anti-MOG and anti-MBP antibodies relapsed more often and earlier than patients without these antibodies. Only 9 of 39 (23\%) antibody seronegative patients suffered a relapse and the mean time to relapse was 45.1 (range 25 to 83) months. In contrast, 21 of $22(95 \%)$ patients with antibodies against both MOG and MBP and 35 of $42(83 \%)$ of patients with only anti-MOG antibodies had a relapse 
Table 1

Antibodies to myelin proteins and some other CNS antigens in MS

\begin{tabular}{lc}
\hline Antigen & Selected reference(s) \\
\hline Myelin basic protein (MBP) & {$[13,36]$} \\
Proteolipid protein (PLP) & {$[45,50]$} \\
Myelin oligodendrocyte glycoprotein (MOG) & {$[2,18,22,29,43,46,51]$} \\
Myelin associated protein (MAG) & {$[6,33]$} \\
Oligodendrocyte-specific protein (OSP) & {$[10]$} \\
2',3'-Cyclic nucleotide 3' phosphodiesterase (CNPase) & {$[49]$} \\
Transaldolase (TAL) & {$[7]$} \\
Gangliosides & {$[1,40]$} \\
Alu repeats & {$[5]$} \\
Neurofilaments (NF) & {$[15,41]$} \\
Proteasome & {$[32]$} \\
Alpha-B-crystallin & {$[3]$} \\
Cerebellar soluble lectin (CSL) & {$[?]$} \\
Heat-shock-protein 60 (hsp 60) & {$[37]$} \\
\hline
\end{tabular}

within a mean of 7.5 (range 1 to $18, P<0.001$ ) and 14.6 (range 2 to $38, P<0.001$ ) months, respectively.

These findings are consistent with previous reports demonstrating that intrathecal IgG antibody production, dominance of B-cells and oligoclonal IgM bands in the CSF are associated with a more progressive disease course $[35,47,48]$.

The reasons for the elevated anti-myelin antibody levels are speculative. It is not known whether these antibodies are biologically active, e.g. demyelinating, or if they merely reflect the extent of tissue damage. Nevertheless these anti-myelin antibodies qualify as measures of disease activity and can therefore be used as a biological marker for disease progression in CIS patients. From a practical point of view it is additionally important that the analysis of anti-myelin antibodies uses rapid and inexpensive methods.

Increased humoral responses against non-myelin antigens are likewise suggested to correlate with disease progression in MS [35]. A promising marker for monitoring axonal damage and therefore for the conversion to chronic progressive MS are neuronal neurofilament proteins. Axonal damage occurs early in the disease course and once a certain threshold of damaged or degenerated axons is reached, secondary progression occurs. Increased levels of antibodies to the light subunit of the neurofilament proteins have been found repeatedly in the primary or secondary chronic progressive phase of the disease course $[15,41]$. Whether these antibodies have a pathogenic role in axonal destruction or are an epiphenomenon secondary to a massive release of axonal proteins remains unclear. Nevertheless these antibodies have shown correlation with clinical disability and brain atrophy in MS.

\section{Future expectations}

It may be anticipated that despite the lack of antibodies for the diagnosis of MS, immunological markers, especially antibodies, may be used in the future to distinguish different immunopathogenetic subtypes of MS. This may support the notion of MS as a heterogeneous disease complex, and in consequence may allow a stratification of patients for individualized immunopathogenetically oriented treatments according to a differential-therapeutic concept.

The publication on the prognostic value of antimyelin antibodies provoked (again) an intense and ongoing discourse on the role and value of antibodies in MS [16,24,28,38]. Subsequently, fruitful cooperations between several research groups were established to provide scientific answers to the above mentioned debate. After validation of the best test system and confirmation of the predictive value of anti-myelin antibodies in other patient cohorts this antibody test may become important for the counseling and management in CIS patients. In particular, antibody analyses may support future decisions on early treatment initiation: those patients at high risk for clinically definite MS should receive immediate early treatment, while patients with low (or even no) risk for a next relapse may see treatment postponed until necessary.

Finally, as the existence of a single antibody that fulfills all the criteria of a surrogate endpoint in MS might prove unlikely, it seems more feasible that in single MS patients a panel of different antibodies, which mirror different stages of inflammation, demyelination, axonal degeneration and remyelination, will need to be characterized in order to optimize therapy and prevent disability. 


\section{References}

[1] N. Achiron, J. Rio, A.L. Fernandez, M. Tintore, I. Duran, I. Galan and X. Montalban, Different antiganglioside antibody pattern between relapsing-remitting and progressive multiple sclerosis, Acta Neurol Scand 93 (1996), 99-103.

[2] M. Adelmann, J. Wood, I. Benzel, P. Fiori, H. Lassmann, J. M. Matthieu, V. Gardinier, K. Dornmair and C. Linington, The N-terminal domain of the myelin oligodendrocyte glycoprotein (MOG) induces acute demyelinating experimental autoimmune encephalomyelitis in the Lewis rat, $J$ Neuroimmunol 63 (1995), 17-27.

[3] M.A. Agius, C.A. Kirvan, A.L. Schafe, E. Gudipati and S. Zhu, High prevalence of anti-alpha-crystallin antibodies in multiple sclerosis: correlation with severity and activity of disease, Acta Neurol Scand 100 (1999), 139-147.

[4] J.J. Archelos, M.K. Storch and H.P. Hartung, The role of B cells and antibodies in multiple sclerosis, Ann Neurol 47 (2000), 694-706.

[5] J.J. Archelos, J. Trotter, S. Previtali, B. Weissbrich, K.V. Toyka and H.P. Hartung, Isolation and characterization of an oligodendrocyte precursor-derived B-cell epitope in multiple sclerosis, Ann Neurol 43 (1998), 15-24.

[6] S. Baig, T. Olsson, J. Yu-Ping, B. Hojeberg, M. Cruz and H. Link, Multiple sclerosis: cells secreting antibodies against myelin- associated glycoprotein are present in cerebrospinal fluid, Scand J Immunol 33 (1991), 73-79.

[7] K. Banki, E. Colombo, F. Sia, D. Halladay, D.H. Mattson, A.H. Tatum, P.T. Massa, P.E. Phillipps and A. Perl, Oligodendrocyte-specific expression and autoantigenicity of transaldolase in multiple sclerosis, J Exp Med 180 (1994), 1649-1663.

[8] T. Berger, Multiple sclerosis, in: Antibodies as Diagnostic and Predictive Markers of Autoimmune Diseases, A.L. Notkins, A. Lernmark and D. Leslie, eds, Autoimmunity 37(4) (2004), 321-324.

[9] T. Berger, P. Rubner, R. Egg, F. Schautzer, H. Ulmer, I. Mayringer, E. Dilitz, F. Deisenhammer and M. Reindl, Antimyelin antibodies as predictors for clinical definite multiple sclerosis in patients with a first demyelinating event, New Engl J Med 146 (2003), 181-197.

[10] J.M. Bronstein, R.L. Lallone, R.S. Seitz, G.W. Ellison and L.W. Myers, A humoral response to oligodendrocyte-specific protein in MS: a potential molecular mimic, Neurology $\mathbf{5 3}$ (1999), 154-161.

[11] W. Brück, K. Neubert, T. Berger and J.R. Weber, Clinical, radiological, immunological and pathological findings in inflammatory CNS demyelination - possible markers for an antibody-mediated process, Multiple Sclerosis 7 (2001), 173177.

[12] A.H. Cross, J.L. Trotter and J.A. Lyons, B cells and antibodies in CNS demyelinating disease, J Neuroimmunol 112 (2001), $1-14$.

[13] M. Cruz, T. Olsson, J. Ernerudh, B. Hojeberg and H. Link, Immunoblot detection of oligoclonal antimyelin basic protein $\mathrm{IgG}$ antibodies in cerebrospinal fluid in multiple sclerosis, Neurology 37 (1987), 1515-1519.

[14] R. Egg, M. Reindl, F. Deisenhammer, C. Linington and T. Berger, Anti-MOG and anti-MBP antibody subclasses in multiple sclerosis, Multiple Sclerosis 7 (2001), 285-289.

[15] R. Ehling, A. Lutterotti, J. Wanschitz, M. Khalil, C. Gneiss, F. Deisenhammer, M. Reindl and T. Berger, Increased frequencies of serum antibodies to neurofilament-light in patients with primary chronic progressive multiple sclerosis, Multiple Sclerosis 10 (2004), 601-606.

[16] S. Gaertner, K.L. de Graa, B. Greve and R. Weissert, Antibodies against glycosylated native MOG are elevated in patients with multiple sclerosis, Neurology 63 (2004), 2381-2383.

[17] C.P. Genain, B. Cannella, S.L. Hauser and C.S. Raine, Identification of autoantibodies associated with myelin damage in multiple sclerosis, Nature Med 5 (1999), 170-175.

[18] M. Hawa, H. Beyan and R.D.G. Leslie, Principles of Autoantibodies as Disease-specific Markers, in: Antibodies as Diagnostic and Predictive Markers of Autoimmune Diseases, A.L. Notkins, A. Lernmark and D. Leslie, eds, Autoimmunity 37(4) (2004), 253-256.

[19] B. Hemmer, J.J. Archelos and H.P. Hartung, New concepts in the immunopathogenesis of multiple sclerosis, Nature Neurosci Rev 3 (2002), 291-301.

[20] E.A. Kabat, M. Glusman and V. Knaub, Quantitative estimation of the albumin and gamma globulin in normal and pathologic cerebrospinal fluid by immunochemical methods, Am J Med 4 (1948), 653-662.

[21] N. Kerlero de Rosbo, R. Milo, M. B. Lees, D. Burger, C.C. Bernard and A. Ben-Nun, Reactivity to myelin antigens in multiple sclerosis. Peripheral blood lymphocytes respond predominantly to myelin oligodendrocyte glycoprotein, J Clin Invest 92 (1993), 2602-2608.

[22] B. Kornek and H. Lassmann, Neuropathology of multiple sclerosis - new concepts, Brain Res Bull 61 (2003), 321-326.

[23] V. Lampasona et al., Similar low frequency of anti-MOG IgG and $\operatorname{IgM}$ in MS patients and healthy subjects, Neurology 62 (2004), 2092-2094.

[24] H. Lassmann, W. Brück and C. Lucchinetti, Heterogeneity of multiple sclerosis pathogenesis: implications for diagnosis and therapy, Trends Mol Med 7 (2001), 115-121.

[25] H. Lassmann, M. Reindl, H. Rauschka, J. Berger, F. AboulEnein, T. Berger, A. Zurbriggen, A. Lutterotti, W. Brück, J.R. Weber, R. Ullrich, M. Schmidbauer, K. Jellinger and M. Vandervelde, A new paraclinical CSF marker for hypoxia-like tissue damage in multiple sclerosis lesions, Brain 126 (2003), $1-11$.

[26] P.V. Lehmann, T. Forsthuber, A. Miller and E.E. Sercarz, Spreading of T-cell autoimmunity to cryptic determinants of an autoantigen, Nature 358 (1992), 155-157.

[27] E.T. Lim, T. Berger, M. Reindl, C.M. Dalton, K. Fernando, G. Keir, E.J. Thompson, D.H. Miller and G. Giovannoni, Antimyelin antibodies do not allow earlier diagnosis of multiple sclerosis, Multiple Sclerosis 11 (2005), 492-494.

[28] C. Linington, M. Bradl, H. Lassmann, C. Brunner and K. Vass, Augmentation of demyelination in rat acute allergic encephalomyelitis by circulating mouse monoclonal antibodies directed against a myelin/oligodendrocyte glycoprotein, Am J Pathol 130 (1988), 443-454.

[29] C.F. Lucchinetti, W. Bruck, M. Rodriguez and H. Lassmann, Distinct patterns of multiple sclerosis pathology indicates heterogeneity on pathogenesis, Brain Pathol 6 (1996), 259-274.

[30] C. Lucchinetti, W. Brück, J. Parisi, B. Scheithauer, M. Rodriguez and H. Lassmann, Heterogeneity of multiple sclerosis lesions: implications for the pathogenesis of demyelination, Ann Neurol 47 (2000), 707-717.

[31] I. Mayo, J. Arribas, P. Villoslada, R. Alvarez Do Forno, S. Rodriguez-Vilarino, X. Montalban, M.R. de Sagarra and J.G. Castano, The proteasome is a major autoantigen in multiple sclerosis, Brain 125 (2002), 2658-2667.

[32] J.R. Moller, D. Johnson, R.O. Brady, W.W. Tourtelotte and R.H. Quarles, Antibodies to myelin-associated glycoprotein 
(MAG) in the cerebrospinal fluid of multiple sclerosis patients, J Neuroimmunol 22 (1989), 55-61.

[33] J.H. Noseworthy, C. Lucchinetti, M. Rodriguez and B.G. Weinshenker, Multiple Sclerosis, New Engl J Med 343 (2000), 938-952.

[34] J.E. Olsson and H. Link, Immunoglobulin abnormalities in multiple sclerosis. Relation to clinical parameters: exacerbations and remission, Arch Neurol 28 (1973), 392-399.

[35] T. Olsson, S. Baig, B. Hojeberg and H. Link, Antimyelin basic protein and antimyelin antibody-producing cells in multiple sclerosis, Ann Neurol 27 (1990), 132-136.

[36] S. Prabhakar, E. Kurien, R.S. Gupta et al., Heat shock protein immunoreactivity in the CSF: correlation with oligoclonal banding and demyelinating disease, Neurology 44 (1994), $1644-1648$.

[37] A.T. Reder and J.J. Oger, Anti-myelin oligodendrocyte glycoprotein antibodies in multiple sclerosis (Editorial), Neurology 62 (2004), 1922-1923.

[38] M. Reindl, C. Linington, U. Brehm, R. Egg, E. Dilitz, F. Deisenhammer, W. Poewe and T. Berger, Antibodies against the myelin oligodendrocyte glycoprotein and the myelin basic protein in multiple sclerosis and other neurological disease: a comparative study, Brain 122 (1999), 2047-2056.

[39] B.T. Sadatipour, J.M. Greer and M.P. Pender, Increased circulating antiganglioside antibodies in primary and secondary progressive multiple sclerosis, Ann Neurol 44 (1998), 980983.

[40] E. Silber, Y.K. Semra, N.A. Gregson and M.K. Sharief, Patients with progressive multiple sclerosis have elevated antibodies to neurofilament subunit, Neurology 58 (2002), 13721381.

[41] M. Sospedra and R. Martin, Immunology of Multiple Sclerosis, Ann Rev Immunol 23 (2005), 683-747.

[42] M.K. Storch, A. Stefferl, U. Brehm, R. Weissert, E. Wallstrom, M. Kerschensteiner, T. Olsson, C. Linington and H. Lassmann, Autoimmunity to myelin oligodendrocyte glycoprotein in rats mimics the spectrum of multiple sclerosis pathology, Brain Pathol 8 (1998), 681-694.
[43] M.K. Storch, S. Piddlesden, M. Haltia, M. Ivanainen, P. Morgan and H. Lassmann, Multiple sclerosis: in situ evidence for antibody- and complement mediated demyelination, Ann Neurol 43 (1998), 465-471.

[44] J.B. Sun, T. Olsson, W.Z. Wang, B.G. Xiao, S. Fredrikson, H.P. Ekre and $\mathrm{H}$. Link, Autoreactive $\mathrm{T}$ and $\mathrm{B}$ cells responding to myelin proteolipid protein in multiple sclerosis and controls, Eur J Immunol 21 (1991), 1461-1468.

[45] J. Sun, H. Link, T. Olsson, B.G. Xiao, G. Andersson, H.P. Ekre, C. Linington and P. Diener, T and B cell responses to myelin-oligodendrocyte glycoprotein in multiple sclerosis, $J$ Immunol 146 (1991), 1490-1493.

[46] L.M. Villar, J. Masjuan, P. Gonzalez-Porque et al., Intrathecal IgM synthesis predicts the onset of new relapses and a worse disease course in MS, Neurology 59 (2002), 555-559.

[47] L.M. Villar, J. Masjuan, P. Gonzalez-Porque, J. Plaza, M.C. Sadaba, E. Roldan, A. Bootello and J.C. Alvarez-Cermeno, Intrathecal IgM synthesis is a prognostic factor in multiple sclerosis, Ann Neurol 53 (2003), 222-226.

[48] M.J. Walsh and J.M. Murray, Dual implication of 2',3'-cyclic nucleotide 3 phosphodiesterase as major autoantigen and C3 complement-binding protein in the pathogenesis of multiple sclerosis, J Clin Invest 101 (1998), 1923-1931.

[49] K.G. Warren and I. Catz, Relative frequency of autoantibodies to myelin basic protein and proteolipidprotein in optic neuritis and multiple sclerosis cerebrospinal fluid, J Neurol Sci $\mathbf{1 2 1}$ (1994), 66-73.

[50] B.G. Xiao, C. Linington and H. Link, Antibodies to myelinoligodendrocyte glycoprotein in cerebrospinal fluid from patients with multiple sclerosis and controls, J Neuroimmunol 31 (1991), 91-96.

[51] J.P. Zanetta, C. Tranchant, S. Kuchler-Bopp et al., Presence of anti-CSL antibodies in the cerebrospinal fluid of patients: a sensitive and specific test in the diagnosis of multiple sclerosis, J Neuroimmunol 52 (1994), 175-182. 


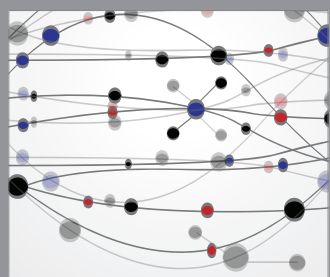

The Scientific World Journal
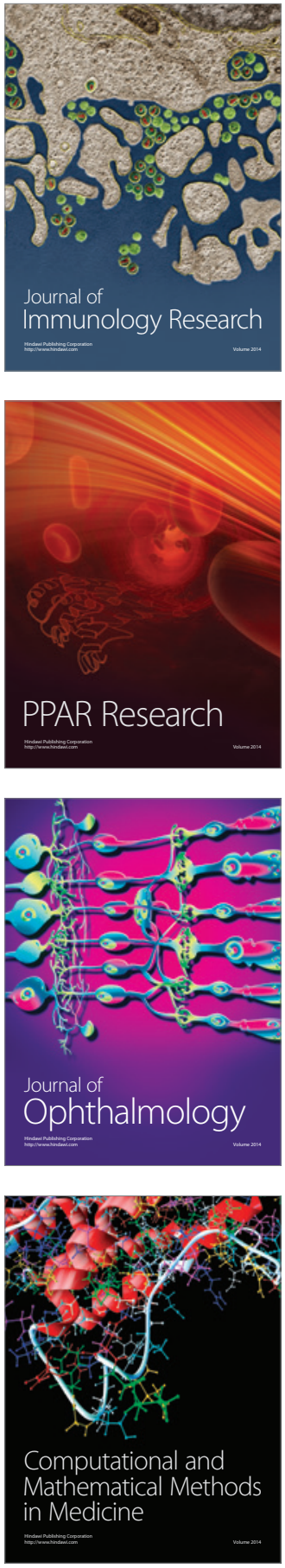

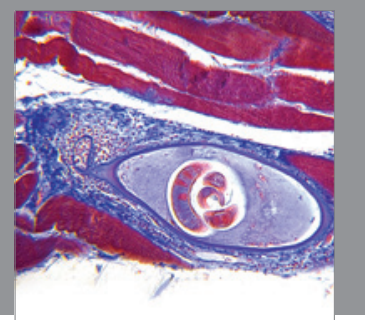

Gastroenterology

Research and Practice
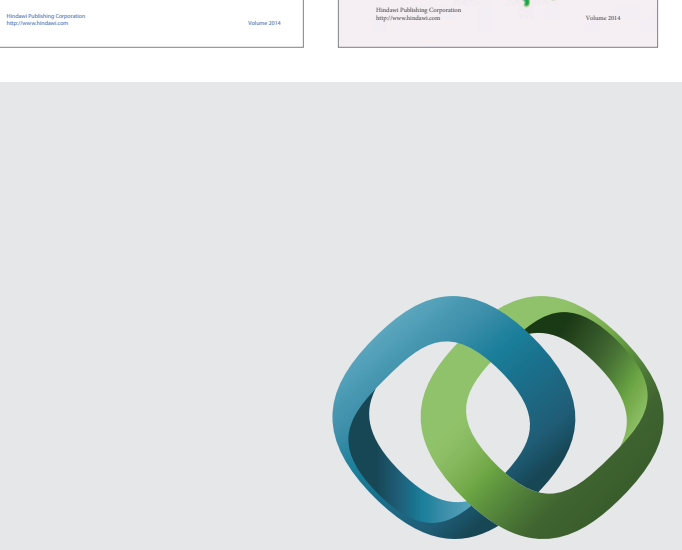

\section{Hindawi}

Submit your manuscripts at

http://www.hindawi.com
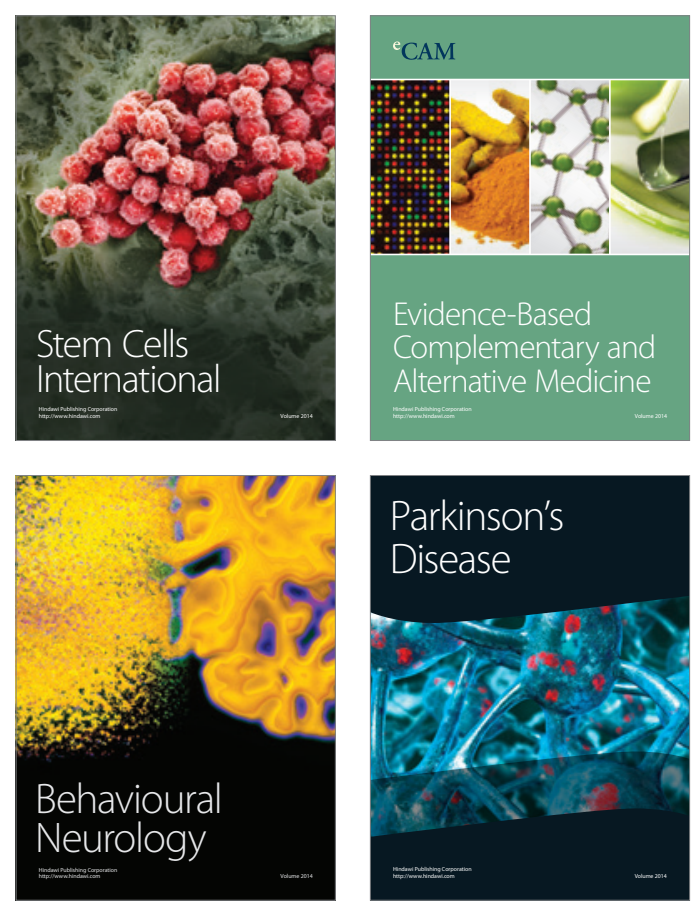

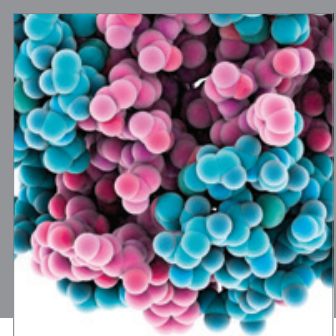

Journal of
Diabetes Research

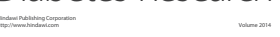

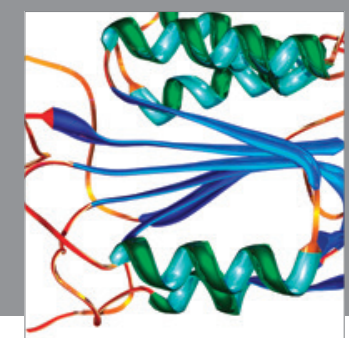

Disease Markers
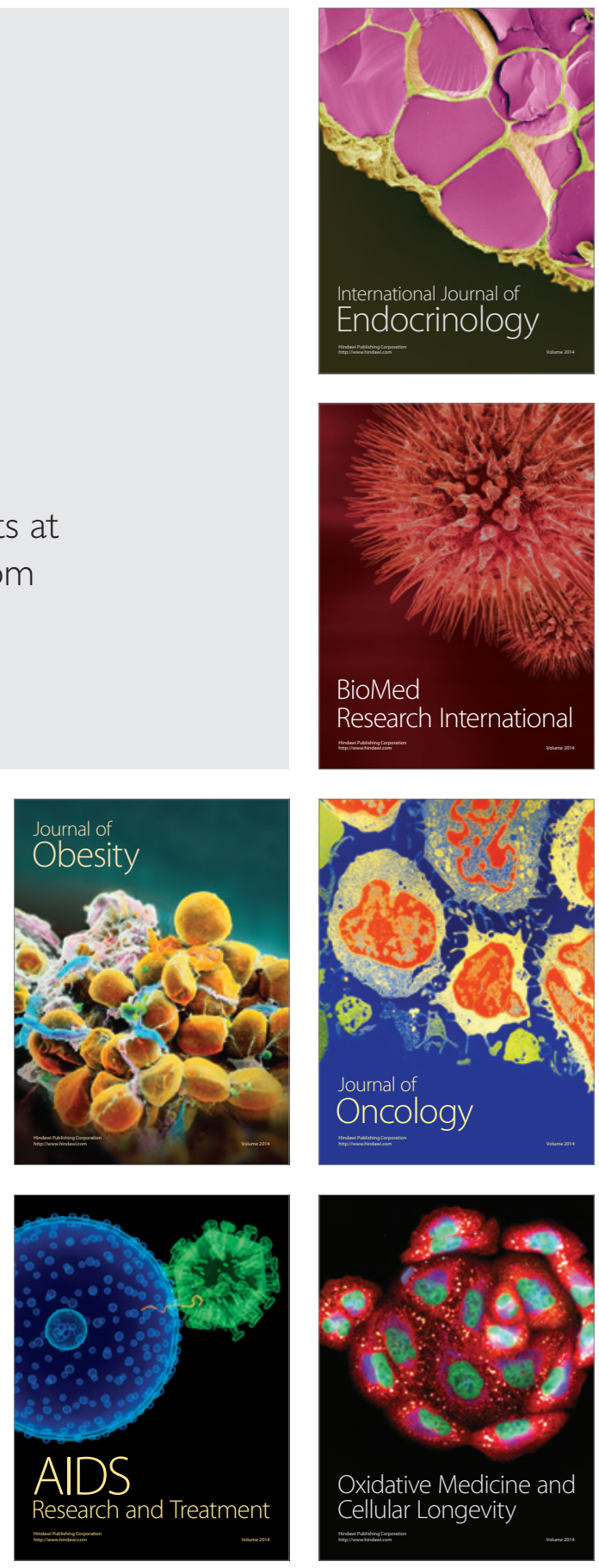